\title{
Building Interoperable Software Components Repository Based on MMF*
}

\author{
Bing Li, Keqing He, Jin Liu, Qiang Wang, Peng Liang, and Rong Peng \\ The State Key Laboratory of Software Engineering (SKLSE), Wuhan University \\ Wuhan, China, 430072 \\ libingyk@public.wh.hb.cn
}

\begin{abstract}
Meta modeling is an effective approach to implement interoperability among many distributed and heterogeneous information sources. A MMF (Meta Model Framework) is a set of Meta objects and Meta modeling constructs to be used in the development a metamodel in the actual implementation of a registry. This paper proposes a common repository model based on MMF to ensure interoperability among heterogeneous software components repositories on the Web. The model will depict what aspects of model elements and constructs we will meet in metamodeling of software components repository. WHCRP (WuHan Component Repository Platform), a prototype system implemented based on MMF, is introduced in the paper.
\end{abstract}

Keywords: MMF (Meta Model framework), software component ontology, software component repository, registry model

\section{Introduction}

An information grid is a software infrastructure using the Grid technologies to achieve integrating, sharing and managing of some heterogeneous information resources scattered across disparate systems on the Web and to provide users or applications with information services on demand. Reusability based on Software component(SC) is an effective approach to implement large-scale software manufacturing production $[1,2]$. A prerequisite for software reuse is a repository that provides functions for classification, storage, management and retrieval of SC [3]. At present, lots of SC repositories, both public and private, already exist today, such as REBOOT [4], STARS [5] and JBCL [6], etc. However, due to difference motivation or difference establisher, those distributed repositories are autonomous and heterogeneous. That is to say, every repository has their own registry model, classification model and terms [6]. On one hand, the diversity of repository is necessary to maintain the ability of a SC repository to application for support concrete domain. On the other hand, it is difficult for a programmer who may be interested in some components stored in many

\footnotetext{
This work was supported by the National Natural Science Foundation of China under Grant 60373086; Wuhan Science \& Technique Key Project under Grant 20021002043; Open Foundation of SKLSE under Grant 03-03; Hubei Provincial Department of Education under Grant 2003A011.
} 
different repositories to access and capture SCs in those repositories. Therefore, it is important to ensure interoperability among SC repositories so as to decrease user's burden of manipulation and increase reuse degree of SC. Software engineering in era of grid computing will depend on a SCs information grid that includes many interoperable SC repositories.

Although many organizations have been in charge of the development of registries standards which will facilitate interoperability such as RIG [7,8], a heterogeneity solution capable of deployment at web scale remains elusive. Using open standards including XML, SOAP, WSDL and UDDI, Web service is becoming a standardized way of integrating Web-based applications. Moreover, Web services use document style messages that offer more flexibility and more pervasiveness than other distributed object specification such as CORBA and DCOM[9]. On one hand, Web service enables reusable SCs to become products marketed on Internet. On the other hand, retrieving SCs is not limited to traditional SC repositories. Because different systems on Web, including traditional SC repositories, ebXML[10] and UDDI [11], describe their SCs with different manners. Registry model heterogeneity will increase rapidly. Then, it is more difficult for SC users to retrieve SCs. A feasible method is to found a common model that presents mapping to registry models of different system. Metamodels define the semantic of modeling elements and constructs that will be used to model the universe of discourse. As a standard draft to present a unified framework for metamodel interoperability MMF (Meta Model Framework) is developed by ISO/IEC/JTC1 in order to establish harmonization of the metamodels, which are developed independently and to reuse them widely across organizations[12].

Based on MMF and ontology for SCs, the paper presents a common SC repository model that achieves a registry framework according to MMF. The paper is organized as follows. In Section 2, we outline the MMF. In Section 3, we describe the SC repository model. In Section 4, we illuminate a prototype implementing the model. Finally, in Section 5, we give our concluding remarks.

\section{Meta-model Framework}

The metamodel framework family of standards consists of a core model of the metamodel framework and a series of metamodel framework, which are to be used in the development of a harmonized metamodel and materialization of the interoperation of existing registries or metamodels. The core metamodel is constructed on MOF (Meta Object Facility) [13] established by OMG(Object Management Group).

\subsection{Meta object Facility}

The heterogeneity in distributed computing environment, Web or grid, needs representation of meta information. In the area of software engineering, metamodel is widely used to describe various models. The MOF Model is referred to as a metametamodel which uses a common abstract syntax for defining metamodels in many typical technologies such as UML, XMI, CORBA, etc. Based on the traditional four layer(M3,M2,M1,M0) metadata architecture, MOF presents a "metadata architec- 


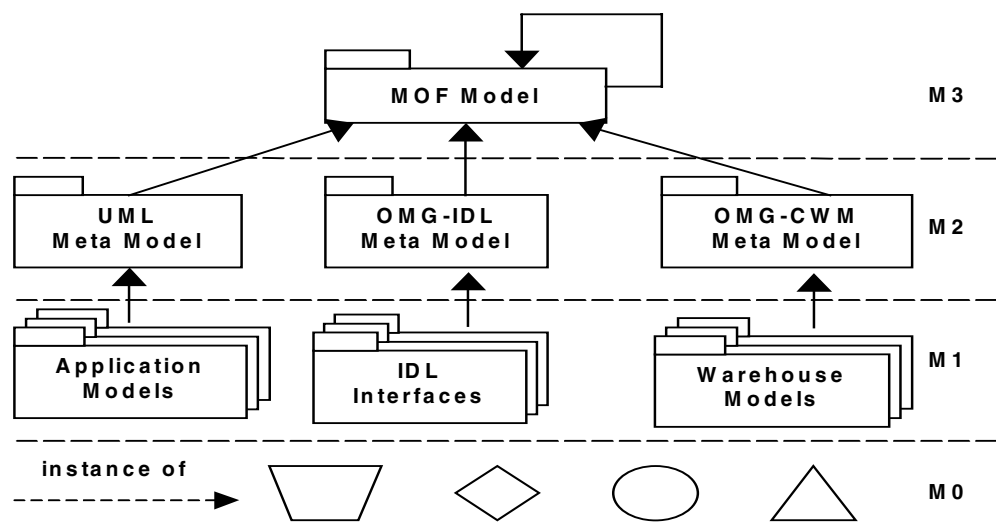

Fig. 1. MOF Metadata Architecture. M3: meta-meta model, M2: metamodel, M1: model, M0:object

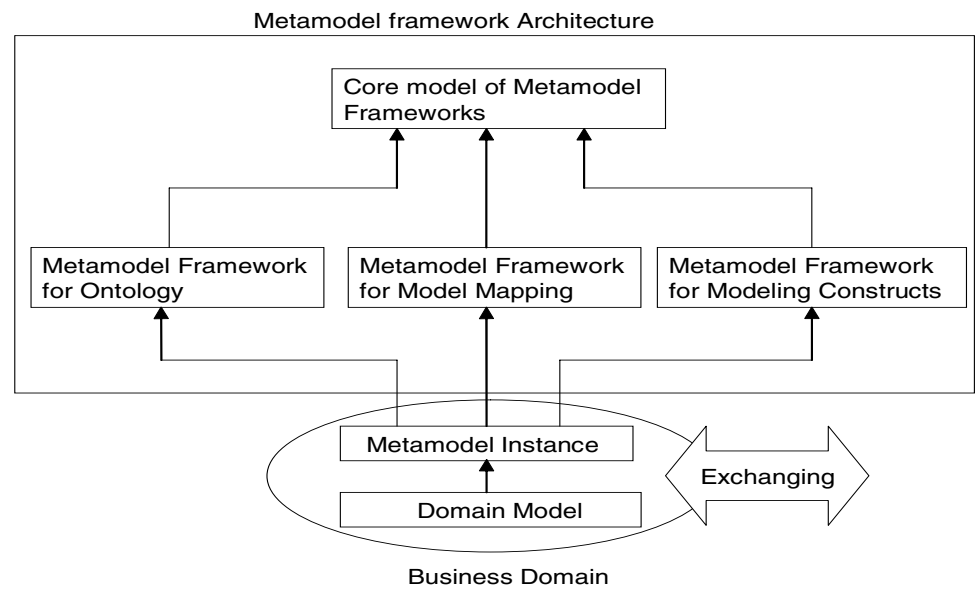

Fig. 2. Architecture of MMF (cited from [14])

ture", illustrated in Figure 1, which defines UML Metamodel, CWM metamodel and other metamodels. Moreover, a mechanism based on MOF/XMI can support interoperability of model information among different platforms.

\subsection{MMF (Meta Model Framework)}

MOF is also the foundation of Meta Model Framework. Currently, MMF architecture consists of four parts: a core metamodel, metamodel framework for ontology, metamodel framework for mapping and metamodel framework for model constructs (See figure 2). However, other useful metamodel frameworks should be proposed in the future [12]. 
In this family of standards, the core model could govern every metamodel framework and they should be developed inheriting concepts and constructs of metamodel frameworks of the core model. The core model should be formulated by inheriting both MOF native metamodels and MDR (ISO/IEC 11179-3) Metamodel, accordingly all of metamodel frameworks have to follow the metamodel concept and basic meta objects of MOF and MDR[14]. The metamodel frameworks in this family of standards should be formulated on UML and MOF.

\section{A Common Model of Software Components Repository}

Based on MMF, we present a common model of SCs repository. Figure 3 illustrates its architecture.

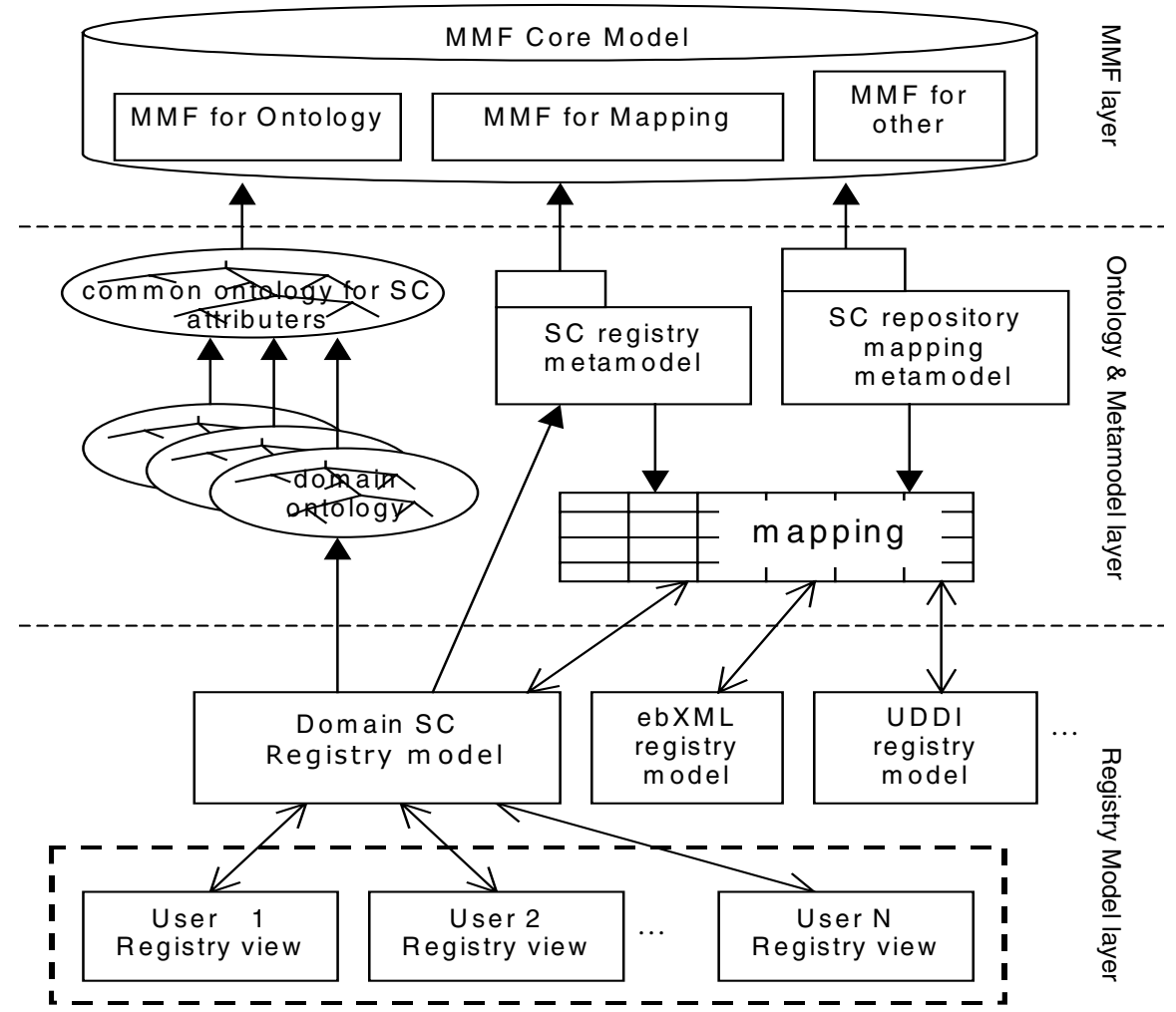

Fig. 3. A Common Model of Software Components Repository

The model consists of three layers. The top layer is MMF including its specifications which ensure the interoperability among heterogeneous software components repositories. The middle layer is Ontology \& Metamodel layer, including SC attribute ontologies, SC registry metamodel and SC repository mapping metamodel. The lowest level is registry model layer. 


\subsection{Software Component Attribute Ontology}

Ontologies provide a shared and consistent understanding of data (and, in some cases, services and processes) that exists within in specific Universe of Discourse(UOD), and how to facilitate communication between people and information systems [15]. Studies have shown that ontologies do great help in many aspects in the information modeling and integrating, such as overcoming semantic heterogeneity among Webbased information systems [16].

Today, various consortia or organization defined schemas with their own manners in the term of the SC attributes. Though inheriting MMF, a common ontology for SC attributes unifies the different concepts and eliminates conflicts existing among registry repository. Furthermore, many typical domain ontologies of SCs could be established for different application environment. Those ontologies embody SCs as an explicit set of concepts, their definitions, attributes and inter-relationships in order to support identifying, classifying and consistency checking in registry.

\subsection{Software Component Registry Metamodel}

MMF is the specification on metamodeling, which is independent from concrete application domains. However, as a application in the area of information classification on Web, SC registry depend on strength metamodel to ensure coherence among SC registry mechanisms in different domains. Governed by MMF, especially MMF for ontology [15], SC registry metamodel not only inherit MMF elements so that SC registry for different applications is easy to understand each other, but also obtain metamodel interoperability between SC registry and non-SC registry. Figure 4 shows a "light-weight" SC registry metamodel we develop.

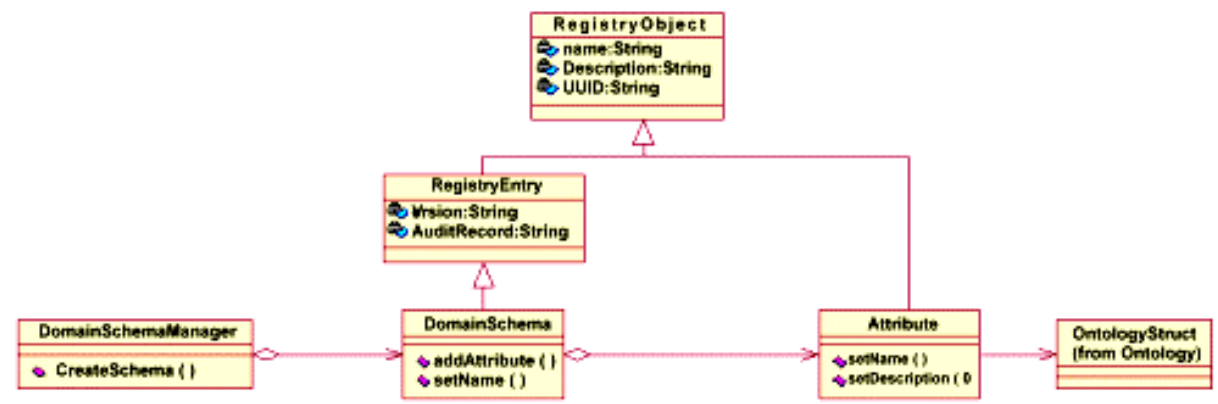

Fig. 4. A SC registry metamodel

\subsection{Registry Model Layer}

The registry model layer of Common Model of SCs Repository includes various registry models. Some registry models developed for special domain are usually stored in special SCs repository. Registry contents of those SCs are different from each other as well as number of registry items. For example, SCs in domain of mobile phone games only have 10 registry items. In contract, SCs in domain of GIS (Geographical Infor- 
mation Systems), e-bank or Web Service might have 50-60 registry items. In addition, some registry models fellow the existed specifications, such as ebXML registry model and UDDI registry model for Web Service. Those registry models are developed not only for SCs but more business objects. Relying on registry metamodel and repository mapping model, it is possible for those registry models achieve interoperability.

\section{Implement of the Common SC Repository Based on MMF}

Based on above models, WHCRP (WuHan Component Repository Platform), a SCs repository prototype is implemented. Figure 5 shows architecture of WHCRP.

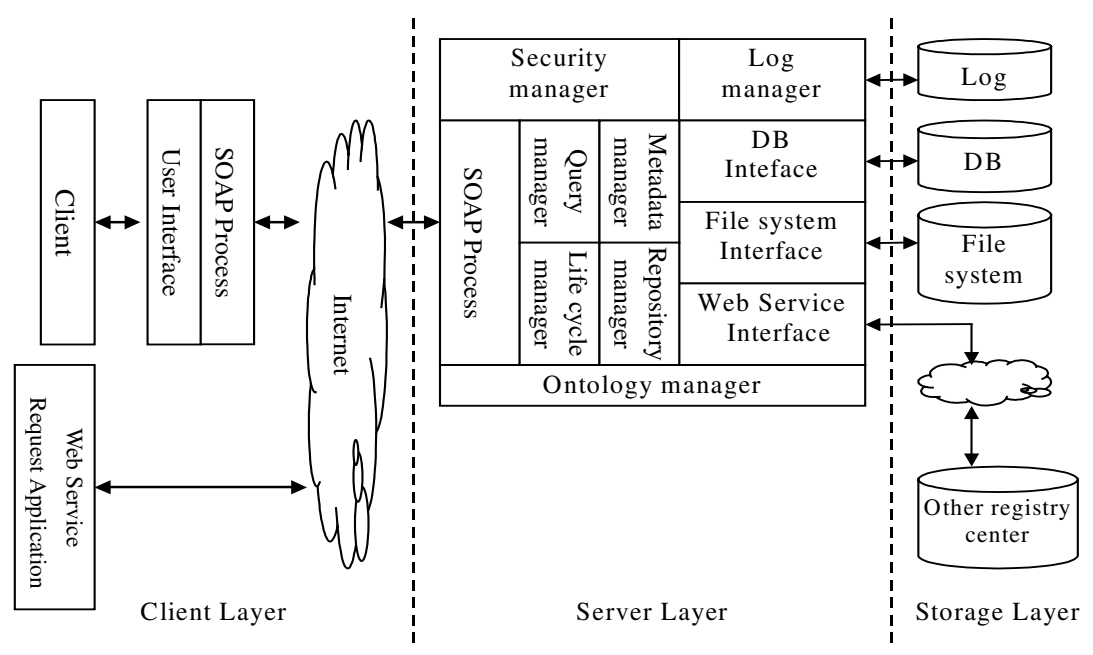

Fig. 5. Architecture of WHCRP

Design and development of the prototype use J2EE (Java 2 Platform, Enterprise Edition) technologies. Functions of each part in the system are explained as follows:

- Client Layer. Client layer consists of user interface and SOAP process. User interface provides access to repository through two views: a customize interface for users and a general Web services API. Because metamodel presents mapping for different registry models, a customize interface means users could adopt a habitual method to register and retrieve SCs. Interaction between client and server uses the Simple Object Access Protocol (SOAP) over HTTP.

- Sever Layer. Main functions of the layer include ontology management, metadata management, query processing, life-cycle management and repository management. There are three kinds of interface adapting to various access storage systems: database, file system and other registry center using Web service also.

- Storage Layer. In our system, there are two kinds of information: meta information of SC registry stored into a database and files of SCs in file system. 


\section{Conclusion}

The objective presented in this paper is relevant with regard to resource interoperability in information grid. Nowadays, reusable Software components on Web are important resource for software engineering. Interoperability among many distributed and heterogeneous SC repositories is regard as a key factor of successful software reuse. MMF (Meta Model Framework) is the framework standard developed by ISO to ensure metamodel interoperability. In this paper, we provide a common SC repository model based on MMF. Combining meta-modeling with ontology, the model enables various registry models, even self-defined registry model to understand each other. By the model, we've built a prototype of SC repository, named WHCRP (WuHan Component Repository Platform), which uses ontology and Web services to create a software component repository platform that offers user-centric support for SCs management and retrieval. The target of our prototype is to achieve transparent access to other SC repositories adopting heterogeneous registry from one repository. Because MMF is yet a working draft, we will fellow its advance and improve our repository model.

\section{References}

1. Alessandro Orso, Mary Jean Harrold, David Rosenblum. Component Metadata for Software Engineering Tasks. Advanced Information Systems Engineering. Proceedings of the $13^{\text {th }}$ International Conference of Advanced Information Systems Engineering (CaiSE2001), 2001

2. Heiko Hahn, Klaus Turowski. Drivers and inhibitors to the development of a software component industry. Proceedings of the $29^{\text {th }}$ EUROMICRO Conference 2003, pp: 128-139, 2003

3. Morel JM, Faget J. The REBOOT Environment. Proceedings of the $2^{\text {nd }}$ International Workshop on Software Reusability Advances in Software. Lucca: IEEE Computer Society Press, 1993. 80-88

4. STARS. Asset Library Open Architecture Framework Version 1.2. Informal Technical Report, START-TC_0404/001/02, August, 1992

5. Keqin Li, Lifeng Guo, Hong Mei, Fuqing Yang. An Overview of JB (Jade Bird) Component Library System JBCL. Proceedings of the $24^{\text {th }}$ International Conference TOOLS ASIA, Beijing, 1997, pp. 261-267

6. Yang Fu-qing,Shao Wei-zhong,MeiHong. The design and implementation of an objectorieted CASE environment in jade bird 2 system. Science in China(Series A), 1995, 25(5):533-542

7. Reuse Interoperability Group (RIG). Basic Interoperability Data Model. Technical Report, RPS0001, Reuse Library Interoperability Group. 1993

8. Reuse Library Interoperability Group. RIG Uniform Data Model for Reuse Libraries (UDM). 1994

9. Jen-Yao Chung, Kwei-Jay Lin, Richard G. Mathieu. Guest Editors' Introduction: Web Services Computing: Advancing Software Interoperability. IEEE Computer, 2003, 36(10): 3537

10. OASIS/ebXML. Registry Information Model v2.0. Approved Committee Specification, http://www.oasis-open.org/committees/regrep/documents/2.0/specs/ebrim.pdf 
11. Universal Description, Discovery and Integration (UDDI). UDDI specifications. http://www.uddi.org/specification.html

12. International Standard Organisation (ISO). Information Technology - Framework for Metamodel Interoperability - Reference Model (Technical report). ISO/IEC 19763-1, Project Editor: Hajime Horiuchi, http://metadata-stds.org/metadata-stds/Document-library/Meeting-reports/SC32WG2 /2003-10-Melbourne/WG2-MEL-047-WD19763-01-MMF-RM.doc

13. Object Management Group (OMG). MOF 1.4 specification. http://www.omg.org/cgi-bin/apps/doc? formal/02-04-03.pdf

14. International Standard Organisation (ISO). Information Technology - Framework for Metamodel Interoperability - Core Model (Technical report). ISO/IEC 19763-2, Project Editor: Masaharu Obayashi, http://metadata-stds.org/metadata-stds/Document-library/Meeting-reports/SC32WG2 /2003-10-Melbourne/WG2-MEL-018-WD19763-2-MMFCore031020.doc

15. International Standard Organisation (ISO). Information Technology - Framework for Metamodel Interoperability - Metamodel Framework for Ontology (Technical report), ISO/IEC 19763-3, Project Editor: KeQing He (SKLSE, China)

16. Guarino N. and Welty C, A Formal Ontology of Properties, In Proceedings of Knowledge Engineering and Knowledge Management: Methods, Models and Tools. Proceedings of $12^{\text {th }}$ International Conference on Knowledge Engineering and Knowledge Management (EKAW2000), Springer Verlag: 97-112, 2000 